\title{
Beating heart surgery via right thoracotomy for reoperative mitral valve surgery: A safe and effective operative alternative
}

\author{
Matthew A. Romano, MD, Jonathan W. Haft, MD, Francis D. Pagani, MD, PhD, and Steven F. Bolling, MD
}

Objective: Right thoracotomy using ventricular fibrillation with cooling has been used for redo mitral valve surgery. This approach avoids the complications of redo sternotomy, such as injury to prior grafts and hemorrhage. As a further refinement, we have used a beating heart technique to further minimize complications while simplifying the operation.

\begin{abstract}
Methods: We reviewed the outcomes of 450 patients who underwent redo mitral valve surgery via a right thoracotomy from 1996 to 2011 at the University of Michigan. Of these, 134 patients underwent redo mitral valve surgery with ventricular fibrillation, and 316 patients underwent beating heart surgery. Although operative eras were consecutive, patients' age, risk factors, New York Heart Association, and preoperative left ventricular ejection fraction were not significantly different. Core temperature on cardiopulmonary bypass for beating heart surgery was $32^{\circ} \mathrm{C}$ versus $26^{\circ} \mathrm{C}$ for ventricular fibrillation.

Results: Patients undergoing beating heart surgery had shorter periods of cardiopulmonary bypass: $81 \pm 9$ minutes versus $113 \pm 36$ minutes. Beating heart surgery required less blood products than ventricular fibrillation: $1.65 \pm 2$ units versus $3.8 \pm 5$ units packed red blood cells, $0.6 \pm 1.2$ units versus $1.8 \pm 4$ units fresh-frozen plasma, and $1.02 \pm 4$ versus $7.5 \pm 17$ platelet packs (all $P<.01$ ). Conversely, patients receiving ventricular fibrillation required longer postoperative ventilation: $34 \pm 101$ hours versus $15.5 \pm 27$ hours $(P<.01)$. The 30 -day mortality was similar for both ( $6.5 \%$ for beating heart and $7.4 \%$ for ventricular fibrillation), and postoperative length of stay was the same at 7 days. Stroke rate was $2.6 \%$ for patients undergoing beating heart surgery and $3 \%$ for patients receiving ventricular fibrillation. Significant operative complications were uncommon; there was no catastrophic hemorrhage, and only 2 patients receiving ventricular fibrillation and 2 patients undergoing beating heart surgery required reexploration.
\end{abstract}

Conclusions: As reoperative cardiac surgery continues to increase, techniques that safely facilitate operation while improving outcome should be adopted. As an operative alternative, redo right thoracotomy mitral valve surgery on the beating heart is associated with shorter bypass time, less transfusion requirements, shorter postoperative ventilation, and lower mortality. This safe and effective approach should be considered for this complex operation. (J Thorac Cardiovasc Surg 2012;144:334-9)

As the population ages, the incidence of reoperative cardiac surgery is projected to continue to increase. Reoperative valve surgery is known to be more complex and associated with increased morbidity and mortality. ${ }^{1,2}$ The right thoracotomy approach with fibrillatory arrest for redo cardiac surgery, especially for atrioventricular valve surgery, is a standard and known alternative to repeat median sternotomy. It avoids complications of repeat sternotomy, including catastrophic reentry into the heart, injury to previous bypass grafts, and significant hemorrhage. ${ }^{3-5}$ Furthermore, right thoracotomy provides excellent exposure to the mitral valve

From the Section of Cardiac Surgery, University of Michigan, Ann Arbor, Mich. Disclosures: Authors have nothing to disclose with regard to commercial support. Received for publication April 20, 2011; revisions received Aug 4, 2011; accepted for publication Sept 21, 2011; available ahead of print Nov 4, 2011.

Address for reprints: Steven F. Bolling, MD, Section of Cardiac Surgery, Cardiovascular Center 5144, 1500 E. Medical Center Dr, Ann Arbor, MI 48109-5864 (E-mail: sbolling@umich.edu).

0022-5223/\$36.00

Copyright $₫ 2012$ Published by Elsevier Inc. on behalf of The American Association for Thoracic Surgery

doi:10.1016/j.jtcvs.2011.09.026 while avoiding extensive mediastinal dissection. Right thoracotomy may also decrease cardiopulmonary bypass (CPB) time, allow for reduced transfusion requirements, and decrease the need for reoperation for bleeding. ${ }^{6-9}$

With this approach, myocardial protection commonly is accomplished with moderate hypothermic fibrillatory arrest (ventricular fibrillation [VF]). We have previously shown, as have others, that this method is often superior to repeat median sternotomy with aortic crossclamping. ${ }^{9-11}$ To further refine the right thoracotomy redo technique, we have used a beating heart (BH) method. We report a retrospective analysis of a single institutional experience of the $\mathrm{BH}$ method for redo mitral valve surgery via a right thoracotomy, highlighting and comparing it with our ventricular fibrillatory arrest experience and data from the Society of Thoracic Surgeons (STS) for redo mitral valve repair in patients with previous sternotomy.

\section{PATIENTS AND METHODS}

From June 1996 to March 2011, 450 patients underwent reoperative mitral valve surgery via right thoracotomy at the University of Michigan 

Abbreviations and Acronyms
$\mathrm{BH}=$ beating heart
$\mathrm{CPB}=$ cardiopulmonary bypass
$\mathrm{ICU}=$ intensive care unit
STS $=$ Society of Thoracic Surgeons
$\mathrm{VF}=$ ventricular fibrillation

Medical Center. Specifically, between 1996 and 1999, 134 patients underwent redo mitral valve surgery via a right thoracotomy with hypothermic ventricular fibrillatory arrest. More recently, from 1999 to 2011, 316 patients underwent mitral valve surgery on the $\mathrm{BH}$ via a right thoracotomy. The preoperative patient demographics for both groups are shown in Table 1 . In the VF group, the mean age was 63 years, $57 \%$ were male, and the mean New York Heart Association class was $2.7 \pm 0.8$. In the $\mathrm{BH}$ group, the mean age was 63 years, $58 \%$ were male, and the New York Heart Association class was $2.6 \pm 0.7$. The mean ejection fraction was $36 \pm 18$ for the VF group and $38 \pm 9$ for the BH group. Previous operations via a sternotomy included coronary artery bypass graft, mitral valve repair, mitral valve replacement, aortic valve replacement, tricuspid valve repair, ventricular septal defect repair, automatic implantable cardiac defibrillator, and atrioventricular canal. Both patient groups had undergone 1 to 4 previous cardiac procedures with a mean of $1.2 \pm 0.6$ procedures in the VF group and $1.4 \pm 0.3$ procedures in the $\mathrm{BH}$ group. The mean time from the last operative procedure to the current procedure was $7 \pm 6$ years for the VF group and $6 \pm 7$ years for the $\mathrm{BH}$ groups. The indication for operation was symptomatic congestive heart failure as described by the American College of Cardiology/American Heart Association guidelines. Preoperatively, all patients were receiving maximal medical therapy for congestive heart failure, including digoxin, diuretics, angiotensinconverting enzyme inhibitors, aldactone, and beta-blockers as tolerated.

All patients had double-lumen endotracheal tubes placed to allow for left lung ventilation while the right lung was decompressed. The patient was positioned in the full right lateral thoracotomy position (left side down), and the groin was prepped and draped to allow for potential femoral arterial cannulation. An external defibrillator was placed before draping for subsequent defibrillation. Preoperative and intraoperative color flow 2-dimensional Doppler transesophageal echocardiography were performed in all patients. Standard intraoperative cardiac monitoring, including arterial and central venous pressure monitoring, and thermodilution pulmonary artery catheterization were used. The chest was entered by a limited lateral thoracotomy incision via the fifth interspace. The pericardium was entered anterior to the phrenic nerve. Arterial cannulation was primarily established via the lateral ascending aorta. When this was not feasible, the right common femoral artery was used for arterial access. Direct bicaval venous cannulation with vacuum assist was used in all patients. Patients receiving fibrillatory arrest were cooled to $26^{\circ} \mathrm{C}$, and fibrillation was induced in $3 \mathrm{pa}-$ tients in whom it was not spontaneously achieved. Patients in the $\mathrm{BH}$ group were allowed to drift to $32^{\circ} \mathrm{C}$. All patients were placed in the Trendelenburg position and rotated to elevate the left atrium relative to the left ventricle. The aorta was vented with an air needle on suction inserted into the ascending aorta, proximal to the arterial cannula. The interatrial groove was generously dissected to expose the left atrium. Mitral valve repair or replacement was performed as determined by the patient's indication or surgeon's preference and was accomplished via a direct left atriotomy. Exposure was facilitated with the use of a self-retaining retractor system (Thompson, Traverse City, Mich). A vent was routinely placed in the right superior pulmonary vein, through a separate stab incision, and passed across into the left inferior pulmonary vein. At the conclusion of the mitral valve repair or bioprosthetic valve replacement, the left ventricular vent was placed across the valve; for mechanical valve replacements, an $8 \mathrm{~F}$
Foley catheter was positioned across the valve to prevent air entrapment while the atrium was still open. $\mathrm{CO}_{2}$ insufflation was not used. Transesophageal echocardiography was used to confirm air removal. Two chest tubes were routinely placed, 1 anterior and 1 posterior. The thoracotomy incision was closed in the standard fashion. When the procedure was complete, the patient was returned to the supine position and the dual lumen endotracheal tube was replaced with a single lumen tube for postoperative ventilation.

End point measures included operative time, CPB time, transfusion requirements, duration of postoperative ventilation, intensive care unit (ICU) stay, postoperative hospital stay, stroke, and hospital mortality. Stroke, as defined by the STS database, is a central neurologic deficit persisting more than 72 hours after surgery. Hospital mortality is defined as inhospital death and 30-day postoperative mortality. Statistical analysis was performed with Statistical Package for the Social Sciences v16 desktop software (SPSS Inc, Chicago, Ill). Continuous data were compared using Student $t$ test or Wilcoxon rank-sum test. All tests were 2 sided.

\section{RESULTS}

Of the 134 patients undergoing redo right thoracotomy with fibrillatory arrest, mitral valve replacement was performed in $55(41 \%)$ and repair was accomplished in the remaining 79. Mitral valve replacement was performed in 101 patients of the $\mathrm{BH}$ group (33\%), and mitral valve repair was performed in 215 patients in the BH group. The decision to replace or repair the valve was made at the time of surgery and dependent on the disease process and operative findings of the native valve or a previously replaced valve. Concomitant operative procedures included patent foramen ovale closure in 20 patients in the VF group and 43 patients in the $\mathrm{BH}$ group, and tricuspid valve repair in 13 patients in the VF group and 72 patients in the $\mathrm{BH}$ group, through a separate right atriotomy. The decision to use femoral cannulation rather than direct aortic cannulation was due to a previously placed ascending aortic graft, location of proximal vein grafts, or calcification of the aortic wall. Femoral cannulation was used in 19 patients in the VF group (14\%) and 28 patients in the $\mathrm{BH}$ group $(9 \%)$.

Patients in the $\mathrm{BH}$ group were in the operating room for a mean time of $187 \pm 62$ minutes, and the patients in the VF group averaged $229 \pm 52$ minutes $(P<.01)$. The CPB times were also shorter for the $\mathrm{BH}$ group. The $\mathrm{BH}$ group averaged $82 \pm 6$ minutes of $\mathrm{CPB}$, compared with $113 \pm 36$ minutes for the VF group $(P<.01)$. Time to extubation was shorter for the $\mathrm{BH}$ group. The length of postoperative mechanical ventilation in the $\mathrm{BH}$ group was $11 \pm 17$ hours compared with $34 \pm 101$ hours in the VF group $(P<.01)$. The BH group also had less transfusion requirements of blood, clotting factors, and platelets. The mean ICU stay was $2.1 \pm 2.0$ days for the $\mathrm{BH}$ group and $2.4 \pm 2.1$ days for the VF group $(P=.45)$. There was no difference in postoperative hospital stay of $7.1 \pm 6.9$ days for the $\mathrm{BH}$ group and $7.3 \pm 5.8$ days for the VF group. The operative and postoperative characteristics are summarized in Table 2.

Overall postoperative complications were low for both groups. Two patients from each group had significant 
TABLE 1. Preoperative characteristics

\begin{tabular}{lcc}
\hline & $\begin{array}{c}\text { Fibrillatory arrest } \\
(\mathbf{1 3 4})\end{array}$ & $\begin{array}{c}\text { Beating heart } \\
(\mathbf{3 1 6})\end{array}$ \\
\hline Age, y & $63 \pm 13$ & $63 \pm 15$ \\
Gender & & \\
$\quad$ Male & 77 & 174 \\
Female & 57 & 143 \\
Mean NYHA functional class & $2.7 \pm 0.8$ & $2.6 \pm 0.7$ \\
Mean ejection fraction (\%) & $36 \pm 13$ & $38 \pm 9$ \\
Previous surgery & & \\
CABG & 97 & 239 \\
Mitral valve repair & 16 & 69 \\
Mitral valve replacement & 10 & 41 \\
Ascending aortic replacement & 1 & 15 \\
Aortic valve replacement & 16 & 48 \\
PFO closure & 4 & 6 \\
Tricuspid valve annuloplasty & 5 & 18 \\
VSD repair & 2 & 1 \\
AV canal repair & 1 & 0 \\
ICD via sternotomy & 3 & 0 \\
No. of previous cardiac operations & $1.2 \pm 0.6$ & $1.4 \pm 0.3$ \\
Time from previous operation (y) & $7 \pm 6$ & $6 \pm 7$ \\
\hline NYHA, New York Heart Association; $C A B G$, coronary artery bypass grafting; $P F O$ \\
patent foramen ovale; $V S D$, ventricular septal defect; $A V$, atrioventricular; $I C D$, im- \\
plantable cardioverter-defibrillator. & & \\
& &
\end{tabular}

postoperative bleeding that required reoperation. Stroke occurred in 8 patients $(2.6 \%)$ in the $\mathrm{BH}$ group and 4 patients $(3 \%)$ in the VF group. Other complications included pleural effusions, hemothorax, pneumothorax, renal failure requiring dialysis, sepsis, pneumonia, and urinary tract infection, none of which differed between groups (Table 3 ). There was 1 intraoperative death in the $\mathrm{BH}$ group from an atrioventricular groove dehiscence. There was no difference in 30-day mortality between groups. There were 22 deaths $(6.9 \%)$ in the $\mathrm{BH}$ group versus 10 patients $(7.4 \%)$ in the VF group $(P=.34)$. In the VF group, cause of death was related to multisystem organ failure in 4 patients, sepsis in 3 patients, cerebrovascular accident in 2 patients, and complications of ischemic colitis in 1 patient. In the BH group, the cause of death was cerebrovascular accident in 5 patients,

TABLE 2. Operative and postoperative characteristics

\begin{tabular}{lccc}
\hline & $\begin{array}{c}\text { Ventricular } \\
\text { fibrillation }\end{array}$ & Beating heart & $\begin{array}{c}\boldsymbol{P} \\
\text { value }\end{array}$ \\
\hline Temperature & $26.2 \pm 2.2$ & $32.6 \pm 1.7$ & $<.01$ \\
Operative time & $229 \pm 52$ & $187 \pm 62$ & $<.01$ \\
CPB time (min) & $113 \pm 36$ & $84 \pm 18$ & $<.01$ \\
PRBC (U) & $3.8 \pm 5$ & $2.8 \pm 3$ & $<.01$ \\
FFP (U) & $1.8 \pm 4$ & $0.7 \pm 1.3$ & $<.01$ \\
Platelets (packs) & $1.5 \pm 3$ & $0.5 \pm 0.8$ & $<.01$ \\
Time to extubation (h) & $34 \pm 101$ & $15 \pm 27$ & $<.01$ \\
Femoral cannulation & 19 & 28 & NS \\
Postoperative ICU stay (d) & $2.4 \pm 2.1$ & $2.1 \pm 2.0$ & NS \\
Postoperative stay (d) & $7.3 \pm 5.8$ & $7.1 \pm 6.9$ & NS \\
\hline
\end{tabular}

$C P B$, Cardiopulmonary bypass; $P R B C$, packed red blood cell; $F F P$, fresh-frozen plasma; $I C U$, intensive care unit; $N S$, not significant.
TABLE 3. Morbidity and mortality characteristics

\begin{tabular}{lcc}
\hline & Fibrillatory arrest & Beating heart \\
\hline Stroke & 4 & 7 \\
Death & 10 & 22 \\
Pleural effusion & 5 & 8 \\
Hemothorax & 2 & 2 \\
Pneumothorax & 3 & 6 \\
Renal failure requiring HD & 2 & 4 \\
Sepsis & 4 & 3 \\
Pneumonia & 2 & 3 \\
Urinary tract infection & 4 & 6 \\
\hline$H D$, Hemodialysis. & &
\end{tabular}

multisystem organ failure in 13 patients, and sepsis from pneumonia in 4 patients.

\section{DISCUSSION}

Right thoracotomy for operation on the mitral valve is not new. It was one of the early approaches to the mitral valve and has been used for more than 60 years. Several previous series have documented its utility and highlighted the advantages in reoperative mitral valve procedures. ${ }^{8-13}$ This single institution series presented further validates and supplements these published reports and results with a BH operative technique. It is known that redo sternotomy usually requires dissection of the aorta and right side of the heart for cannulation and access to the mitral valve, and frequently dissection of the left side to permit the heart to rotate in the pericardium to improve visualization of the mitral valve when opening the left atrium. Such extensive dissection increases the risk of bleeding and injury to venous and arterial bypass grafts. A right thoracotomy facilitates efficient exposure to the mitral valve while avoiding extensive mediastinal dissection, bypass grafts, and the anterior heart, which are not in the operative field.

VF with moderate to deep hypothermia traditionally provided for myocardial protection and was used with a right thoracotomy. Early in our experience, we used a similar technique. To further simplify intervention on this complex patient population, we adopted a warmer $\mathrm{BH}$ approach, minimizing hypothermia and avoiding fibrillatory arrest. This BH approach obviates the need for deeper hypothermia because of subendocardial hypoperfusion mismatches, which are commonly seen with VF. During electrically induced fibrillation, oxygen delivery to the left ventricle is markedly reduced and coronary flow is redistributed away from the subendocardium. ${ }^{14}$ By keeping the heart in its natural beating state with antegrade coronary flow, the risk of reperfusion injury is potentially mitigated.

Also during ischemic arrest, myocardial edema increases in the static diastolic state and may cause cardiac dysfunction. By keeping the heart beating, myocardial edema is decreased and function may be maintained, which may be of 
particular importance in these patients with already impaired ventricular function. ${ }^{14}$ In this series of patients undergoing BH surgery, myocardial protection was excellent and failure to wean from CPB was not noted. Others have also suggested that warm BH surgery may also provide enhanced myocardial protection and decrease reperfusion insult.

Previous reports have cited less transfusion requirements for reoperative mitral valve surgery via the right chest compared with median sternotomy. ${ }^{14,15}$ In our series, patients in the $\mathrm{BH}$ group were not actively cooled but were allowed to drift to approximately $32^{\circ} \mathrm{C}$, and the heart was left beating. These patients were noted to have significantly lower transfusion requirements, almost routinely requiring half the amount of blood products. We acknowledge that, over time, emerging technologies and techniques certainly could have played a role in decreased coagulopathy. Nonetheless, it would appear that the warmer core temperatures decrease hypothermic-mediated coagulopathy.

Engelman and colleagues ${ }^{16}$ showed fibrinolysis was significantly decreased in patients undergoing cardiac surgery if CPB was maintained at $32^{\circ} \mathrm{C}$. The decrease in the formation of plasmin was significantly different than emergence from cold CPB. The warmer core temperature can be extrapolated to shorter $\mathrm{CPB}$ duration. In our series, CPB was significantly shorter for $\mathrm{BH}$ than VF. A BH technique eliminates the additional time required for cooling and rewarming for VF. The added benefit of shorter periods of CPB may also be responsible for less transfusion requirements. Although the use of aprotinin in patients undergoing redo operations during some of this era may have played a role in the overall lower incidence of bleeding, it was balanced between groups and not used in the later years of $\mathrm{BH}$ surgery, when in fact no rebound increase in bleeding rate has been noted. Finally, the decision to replace or repair the mitral valve was dependent on the operative findings or a previously replaced valve. There was a higher mitral valve repair rate in the $\mathrm{BH}$ series than in $\mathrm{VF}$, which may have influenced CPB time and therefore complications.

We also noted shorter time periods of mechanical ventilation in the $\mathrm{BH}$ postoperative period. It has been suggested that a right thoracotomy may compromise pulmonary function especially when compared with repeat median sternotomy. ${ }^{8}$ Pleuro-pulmonary complications were not significant in our series. Our overall time of postoperative ventilation was not excessively long. Perhaps the lower transfusion requirements indicate less hemodynamic instability, favoring extubation criterion. We have well-established postoperative mechanical ventilation and pulmonary protocols that have evolved over time. Postoperative mechanical ventilation time of 34 hours for VF and 15 hours for BH in our series compares favorably to the STS national database mean of 58 hours of postoperative ventilation for reoperative mitral valve surgery. ${ }^{17}$
We were able to establish central cannulation for CPB almost routinely. It has been suggested that a right thoracotomy necessitates peripheral cannulation and may be associated with increased cerebral vascular accidents. ${ }^{18}$ The lateral ascending aorta and superior and inferior venae cavae are readily accessible. The factors that tended to lead to femoral cannulation were previously placed prosthetic ascending aortic grafts, aberrant proximal venous bypass graft location, or calcified aortic wall. Femoral cannulation was required in only $10.4 \%$ (47) of the total series of 450 patients, and there was no difference between the $\mathrm{VF}$ and $\mathrm{BH}$ groups, with less in the later part of the series.

It is well known that the right thoracotomy offers excellent exposure to the mitral valve. The surgeon is afforded the luxury of looking directly down on the valve through the left atriotomy. Also, the $\mathrm{BH}$ approach enables the assessment of the function and geometry of the valve (Figure 1) and subsequently accurately facilitates repair throughout the operation, which is not possible during static fibrillation. ${ }^{19}$

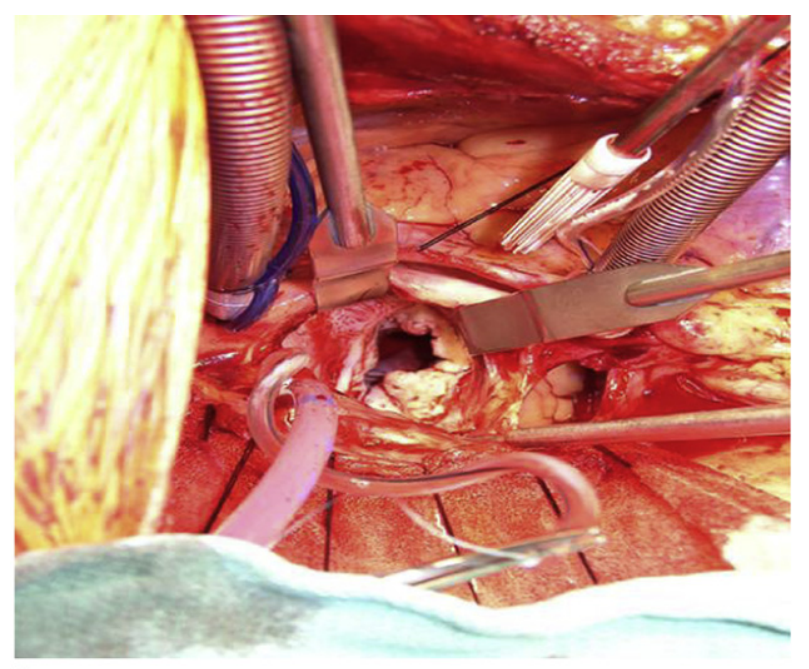

A

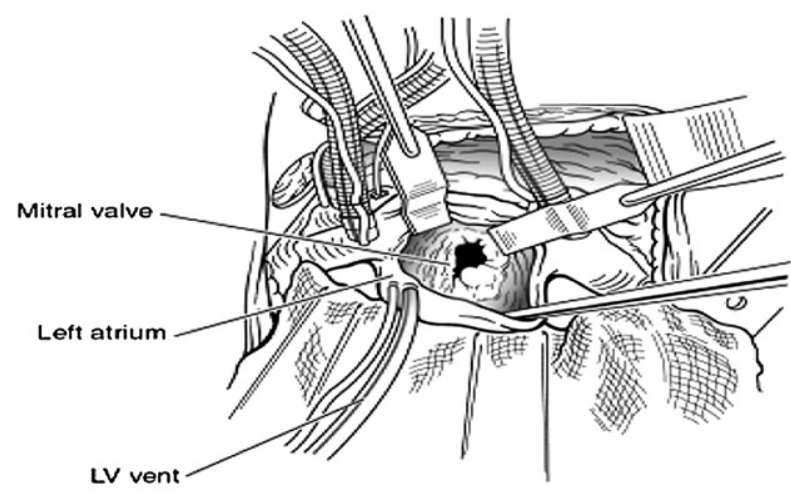

B

FIGURE 1. Intraoperative view of BH redo mitral valve surgery: Photograph (A) and sketch (B) of a third-time redo with intact thoracic and saphenous grafts. $L V$, Left ventricle. 
TABLE 4. Comparison with Society of Thoracic Surgeons' National Database for reoperative isolated mitral valve surgery

\begin{tabular}{lccc}
\hline & $\begin{array}{c}\text { Fibrillatory } \\
\text { arrest }\end{array}$ & $\begin{array}{c}\text { Beating } \\
\text { heart }\end{array}$ & $\begin{array}{c}\text { STS National } \\
\text { Database }\end{array}$ \\
\hline Age & 63 & 65 & 63 \\
Mean ejection fraction & 36 & 38 & 49 \\
Postoperative ICU stay (d) & 2.4 & 2.1 & 4.9 \\
Postoperative stay (d) & 7.3 & 7.1 & 11.1 \\
Time to extubation (h) & 34 & 11 & 58 \\
CPB time (min) & 113 & 82 & 134 \\
Reoperation for bleeding (\%) & 1.5 & 0.6 & 5 \\
Stroke (\%) & 3 & 2.6 & 3 \\
Mortality (\%) & 7.4 & 6.9 & 8.2 \\
\hline STS, Society of Thoracic Surgeons. & & &
\end{tabular}

STS, Society of Thoracic Surgeons.

All right thoracotomy CPB series stress the importance of meticulous technique to ensure complete removal of intracardiac air. We routinely use transesophageal echocardiography to assess the competence of the mitral and aortic valves and to evaluate air removal. In addition, the patient is placed in the Trendelenburg position before opening the heart, an air evacuation needle is placed in the ascending aorta, and the air in the left ventricle is evacuated by making the mitral valve incompetent with a vent, thereby preventing the unloaded left ventricle from ejecting air. A recent series from the Cleveland Clinic surmised that the right thoracotomy increased the risk of mitral valve reoperation and was associated with an increase in stroke. However, in that report by Svensson and colleagues, ${ }^{18}$ of 80 patients undergoing right thoracotomy redo mitral valve surgery (of 2469 redo surgeries), crossclamping of the aorta was frequently used, as was hypothermic circulatory arrest, and femoral cannulation was used almost exclusively. Their overall mortality was $6.3 \%$, similar to this series of 450 patients, but their stroke rate was high at $7.5 \%$, versus $2.6 \%$ in this $\mathrm{BH}$ series. We agree with their findings that right thoracotomy with VF and femoral cannulation may be suboptimal. Direct aortic cannulation and a warm $\mathrm{BH}$ technique are associated with a lower incidence of stroke. To further compare our findings, we used the STS national database as a reference for reoperative isolated mitral valve surgery (Table 4). The BH group presented had shorter CPB time, mechanical ventilation time, and postoperative ICU and hospital stay. Furthermore, although statistical analysis is not possible, mortality and stroke seem favorable with a BH approach.

Finally, there are several considerations that may preclude redo right thoracotomy for mitral valve repair, and these also pertain to the warm BH method. Briefly, simultaneous coronary artery bypass grafting will require exposure that may be accomplished only with median sternotomy. Significant aortic insufficiency would be a contraindication, and aortic valve replacement would be indicated. Consideration should be given to patients with significant pulmonary pathology or respiratory insufficiency.

\section{CONCLUSIONS}

We acknowledge the limitations of this retrospective, observational operative technique study and conclusions that may be drawn. Furthermore, well-established inherent patient factors certainly determine long-term outcome. Nonetheless, the data presented show that in appropriately selected patients, reoperative mitral valve surgery via a right thoracotomy on the $\mathrm{BH}$ is a safe, effective, and reliable procedure that simplifies the operative approach. Difficult mediastinal dissection is avoided, and excellent exposure and evaluation of the abnormal valve pathology and subsequent repair or replacement of the mitral valve are afforded to the operating surgeon. The associated shorter bypass and operative times, lower transfusion requirements, and shorter postoperative mechanical ventilation time, with acceptable low morbidity and mortality, compare favorably to national averages for reoperative mitral valve surgery. BH surgery via right thoracotomy for reoperative mitral valve surgery is a safe and effective alternative for cardiac surgeons to consider for their patients.

\section{References}

1. Antunes MJ. Techniques of valvular reoperation. Eur J Cardiothorac Surg. 1992; 6(Suppl 1):554-8.

2. Hannan EL, Kilburn H Jr, O'Donnell JF, Lukacik G, Shields EP. Adult open heart surgery in New York state: an analysis of risk factors and hospital mortality rates. J Am Med Assoc. 1990;264:2768-74.

3. Macmanus Q, Okies JE, Phillips SJ, Starr A. Surgical considerations in patients undergoing repeat median sternotomy. J Thorac Cardiovasc Surg. 1975;69: 138-43.

4. Dobell AR, Jain AK. Catastrophic hemorrhage during redo sternotomy. Ann Thorac Surg. 1984;37:273-8.

5. Loop FD. Catastrophic hemorrhage during sternal reentry. Ann Thorac Surg. 1984;37:271-2.

6. Berreklouw E, Alfieri O. Revival of right thoracotomy to approach atrioventricular valves in reoperations. Thorac Cardiovasc Surg. 1984;32:331-3.

7. Gillinov AM, Casselman FP, Lytle BW, Blackstone EH, Parsons EM, Loop FD, et al. Injury to a patent left internal thoracic artery graft at coronary reoperation. Ann Thorac Surg. 1999;67:382-6.

8. Tribble CG, Killinger WA Jr, Harman PK, Crosby IK, Nolan SP, Kron IL. Anterolateral thoracotomy as an alternative to repeat median sternotomy for replacement of the mitral valve. Ann Thorac Surg. 1987;43:380-2.

9. Byrne JG, Karavas AN, Adams DH, Aklog L, Aranki SF, Filsoufi F, et al. The preferred approach for mitral valve surgery after CABG: right thoracotomy, hypothermia and avoidance of LIMA-LAD graft. J Heart Valve Dis. 2001;10: 584-90.

10. Steimle CN, Bolling SF. Outcome of reoperative valve surgery via right thoracotomy. Circulation. 1996;94(9 Suppl):II126-8.

11. Holman WL, Goldberg SP, Early LJ, McGiffin DC, Kirklin JK, Cho DH, et al. Right thoracotomy for mitral reoperation: analysis of technique and outcome. Ann Thorac Surg. 2000;70:1970-3.

12. Byrne JG, Aranki SF, Adams DH, Rizzo RJ, Couper GS, Cohn LH. Mitral valve surgery after previous CABG with functioning IMA grafts. Ann Thorac Surg. 1999;68:2243-7.

13. Cohn LH, Peigh PS, Sell J, DiSesa VJ. Right thoracotomy, femorofemoral bypass, and deep hypothermia for replacement of the mitral valve. Ann Thorac Surg. 1989;48:69-71.

14. Thompson MJ, Behranwala A, Campanella C, Walker WS, Cameron EWJ. Immediate and long-term results of mitral prosthetic replacement using a right thoracotomy beating heart technique. Eur J Cardiothorac Surg. 2003;24: 47-51.

15. Braxton JH, Higgins RS, Schwann TA, Sanchez JA, Dewar JL, Kopf GS, et al. Reoperative mitral valve surgery via right thoracotomy: decreased blood loss and improved haemodynamics. J Heart Valve Dis. 1996;5:169-73. 
16. Engelman R, Pleet A, Rousou J, Flack J, Deaton D, Gregory C, et al. What is the best perfusion temperature for coronary revascularization? J Thorac Cardiovasc Surg. 1996;112:1622-33.

17. Lee R, Li S, Rankin JS, O'Brien SM, Gammie JS, Peterson ED, et al. Society of Thoracic Surgeons Adult Cardiac Surgical Database. Fifteen year outcome trends of valve surgery in North America. Ann Thorac Surg. 2011;91:677-84.
18. Matsumoto Y, Watanabe G, Endo M, Sasaki H, Kasashima F, Kosugi I. Efficacy and safety of on-pump beating heart surgery for valvular disease. Ann Thorac Surg. 2002;74:678-83.

19. Svensson LG, Gillinov AM, Blackstone EH, Houghtaling PL, Kim KH, Pettersson GB, et al. Does right thoracotomy increase the risk of mitral valve reoperation? J Thorac Cardiovasc Surg. 2007;134:677-82. 